\title{
Direct multi-wavelength limb-darkening measurements of three late-type giants with the Navy Prototype Optical Interferometer
}

\author{
M. Wittkowski ${ }^{1 \star}$, C. A. Hummel ${ }^{2}$, K. J. Johnston ${ }^{2}$, D. Mozurkewich ${ }^{3}$, A. R. Hajian ${ }^{2}$, and N. M. White ${ }^{4}$ \\ 1 Universities Space Research Association (USRA), 300 D Street SW, Suite 801, Washington, DC 20024, USA \\ Mailing address: U.S. Naval Observatory, AD5, 3450 Massachusetts Avenue NW, Washington, DC 20392, USA \\ 2 U.S. Naval Observatory, 3450 Massachusetts Avenue NW, Washington, DC 20392, USA \\ e-mail: cah@usno.navy.mil, kjj@astro.usno.navy.mil, hajian@usno.navy.mil \\ 3 Naval Research Laboratory, Code 7210, Washington, DC 20375, USA \\ e-mail: mozurk@rsd.nrl.navy.mil \\ 4 Lowell Observatory, 1400 West Mars Hill Road, Flagstaff, AZ 86001, USA \\ e-mail: nmw@lowell.edu
}

Received 22 January 2001 / Accepted 9 August 2001

\begin{abstract}
We present direct measurements of the limb-darkened intensity profiles of the late-type giant stars HR 5299, HR 7635, and HR 8621 obtained with the Navy Prototype Optical Interferometer (NPOI) at the Lowell Observatory. A triangle of baselines with lengths of $18.9 \mathrm{~m}, 22.2 \mathrm{~m}$, and $37.5 \mathrm{~m}$ was used. We utilized squared visibility amplitudes beyond the first minimum, as well as triple amplitudes and phases in up to 10 spectral channels covering a wavelength range of $\sim 650 \mathrm{~nm}$ to $\sim 850 \mathrm{~nm}$. We find that our data can best be described by featureless symmetric limb-darkened disk models while uniform disk and fully darkened disk models can be rejected. We derive high-precision angular limb-darkened diameters for the three stars of 7.44 mas \pm 0.11 mas, 6.18 mas \pm 0.07 mas, and 6.94 mas \pm 0.12 mas, respectively. Using the HIPPARCOS parallaxes, we determine linear limb-darkened radii of $114 R_{\odot} \pm 13 R_{\odot}, 56 R_{\odot} \pm 4 R_{\odot}$, and $98 R_{\odot} \pm 9 R_{\odot}$, respectively. We compare our data to a grid of Kurucz stellar model atmospheres, with them derive the effective temperatures and surface gravities without additional information, and find agreement with independent estimates derived from empirical calibrations and bolometric fluxes. This confirms the consistency of model predictions and direct observations of the limb-darkening effect.
\end{abstract}

Key words. techniques: interferometric - techniques: high angular resolution - stars: fundamental parameters stars: atmospheres - stars: late-type

\section{Introduction}

For a detailed understanding of stellar atmospheres and stellar evolution, it is of essential importance to obtain accurate observational estimates of stellar surface structure parameters on all scales. These parameters include diameters, limb-darkening profiles, photospheric asymmetries, and special features like hot spots. Model atmospheres are mainly constrained by observations of stellar spectra. Direct measurements of the limb-darkening profile can, in principle, provide an independent estimate of the temperature change with continuum opacity and, thus, an independent observational verification. So far, the detailed

Send offprint requests to: M. Wittkowski,

e-mail: mwittkow@eso.org

* Present address: European Southern Observatory, Casilla 19001, Santiago 19, Chile. intensity profile and the whole variety of additional surface structure parameters could only be observed in the case of the Sun. Today's interest, however, includes other phases of stellar structure and evolution, for instance stars in late evolutionary phases (Manduca et al. 1977; Scholz \& Takeda 1987; Hofmann \& Scholz 1998; Jacob et al. 2000). These stars might exhibit asymmetric (e.g. Wittkowski et al. 1998) and even highly fragmented (e.g. Weigelt et al. 1998) mass-loss events, which are believed to be triggered by the conditions on the stellar surfaces. Unfortunately, direct measurements of surface structure parameters are rare. While diameters have so far been obtained for several hundred stars with interferometric and lunar occultation techniques, the second-order effect, limb-darkening, has been directly observed for only a very limited number of stars (Hanbury Brown et al. 1974; Haniff et al. 1995; Quirrenbach et al. 1996; Hajian et al. 1998). Additional 
surface features have been detected on the apparently largest supergiants $\alpha$ Ori, $\alpha$ Sco, and $\alpha$ Her contributing with $5 \%$ to $20 \%$ to the total source fluxes at optical wavelengths (Buscher et al. 1990; Wilson et al. 1992; Gilliland \& Dupree 1996; Burns et al. 1997; Tuthill et al. 1997; Young et al. 2000).

The NPOI, located near Flagstaff, Arizona, is especially designed for imaging of stars and their environments and is described in detail by Armstrong et al. (1998). The methods of "baseline bootstrapping" and "wavelength bootstrapping" (see Roddier 1988; Quirrenbach et al. 1996; Hajian et al. 1998) can be used in order to detect weak fringe contrasts, i.e. low visibility values, on resolved stars by detecting the higher-contrast fringes on a chain of shorter effective spacings which comprise the long baselines. Hajian et al. (1998) demonstrated that bootstrapping with the NPOI astrometric subarray enabled the measurement of visibility values of a resolved stellar disk beyond the first minimum. By analyzing NPOI triple visibility products, they verified that the intensity profiles of $\alpha$ Ari and $\alpha$ Cas deviate from uniform disks due to the effect of limb-darkening.

Here, we use NPOI's bootstrapping ability and apply an improved bias correction in order to utilize squared visibility amplitudes, in addition to triple amplitudes and closure phases, of three much fainter but well resolved latetype stars for spatial frequencies on both sides of the first minimum. Since there are three squared visibility amplitudes for each triple amplitude, more information can be used for the analysis of stellar intensity profiles. In an attempt to check the consistency of direct observations and model predictions of the limb-darkening effect as a function of continuum wavelength, effective temperature, and surface gravity, we compare our multi-wavelength interferometric data to a grid of Kurucz stellar model atmospheres. In addition, we determine high-precision limbdarkened diameters.

\section{Observations}

Interferometric observations of the three late-type giants, HR 5299 (M4), HR 7635 (K5), and HR 8621 (M4), were performed with the NPOI using the configuration described by Benson et al. (1997).

The center, east, and west siderostats of the astrometric subarray were used with effective apertures of $12.5 \mathrm{~cm}$. They provide baselines with lengths of $18.9 \mathrm{~m}, 22.2 \mathrm{~m}$, and $37.5 \mathrm{~m}$ at azimuths (measured east from north) of $-67.5,63.6$, and 86.0 , respectively. The three afocal beams of light are reduced in diameter and sent into vacuum delay-lines for compensation of optical path differences (OPDs) before they are combined pairwise using beamsplitters. Three of the afocal output beams corresponding to the three baselines are dispersed by a prism, focused by a lenslet array onto 32 optical fibers, and detected by avalanche photodiodes (APDs), covering a spectral range from $450 \mathrm{~nm}$ to $850 \mathrm{~nm}$. The fringe packet is detected through modulation of the OPDs and is kept centered close to zero residual delay using the method of "group delay fringe tracking" (Armstrong et al. 1998).

Table 1 lists names and characteristics of the observed stars together with the observing dates, the number of obtained scans, and the names and estimated diameters of the calibrator stars. During a scan of $90 \mathrm{~s}$, the photon count rate for every channel is determined in eight temporal bins (synchronous with the delay line modulation), which sample a fringe every $2 \mathrm{~ms}$. After each scan, a background measurement was taken on blank sky near the star. Immediately before or after each scan of a program star, a scan on one of the calibrator stars as specified in Table 1 was recorded. The calibrator stars were chosen to be located near the appropriate program stars on the sky. Their diameters were estimated using a calibration obtained by Mozurkewich et al. (1991) based on the apparent visual magnitude and the $(R-I)$ color index and are small enough so that possible errors in this estimate do not noticeably affect the calibration of our much larger resolved program stars.

In order to compensate for detection noise bias terms (see Sect. 3), incoherent (i.e. fringeless) data on several stars covering a range of apparent visual magnitudes were recorded on July 22, 2000, by moving the delay lines off the fringe packet.

The signal-to-noise ratio of the measured visibilities decreases for spectral channels with shorter wavelengths owing to their narrower bandwidths (the channels are equally spaced in wavenumber), poorer seeing, and the red color of the observed stars. Therefore, only the 10 reddest channels were used for the data analysis of HR 7635, and the 5 reddest channels for that of the fainter stars HR 5299 and HR 8621. The central wavelengths of the 10 reddest spectral channels are known to within about $1 \%$ to be $(852,822,794,769,745,723,702,683$, $665,649) \mathrm{nm}$, while their bandwidths range from $\sim 31 \mathrm{~nm}$ for the $852 \mathrm{~nm}$ channel to $\sim 16 \mathrm{~nm}$ for the $649 \mathrm{~nm}$ channel. These passbands mainly provide continuum observations and are not dominated by spectral features. The absorption band/continuum ratio is relatively small for the K5 star and the strong TiO bands at $671 \mathrm{~nm}$ and $714 \mathrm{~nm}$ are not covered by the spectral channels used for the M 4 stars. However, for the M 4 giants, some spectral channels are affected by molecular absorption bands, which will be taken into account.

Figure 1 shows the obtained coverages of the $u v$-plane for HR 5299, HR 7635, and HR 8621 based on all observation dates and those spectral channels used for the data analysis. These spatial frequencies range up to a radius of $\sim 300$ cycles/arcsec, which corresponds to a spatial resolution of 3.3 mas.

\section{Data reduction and calibration}

The raw data were processed, reduced, and calibrated as described in detail by Hummel et al. (1998). This process includes (1) calculation of the real $\left(X_{i}\right)$ and imaginary $\left(Y_{i}\right)$ parts of the complex visibility for each baseline $i$ and each 
Table 1. Names (bright star catalog number HR, FK5 catalog number, common name) of the program stars, together with their properties (spectral type, apparent visual magnitude $m_{\mathrm{V}}$, HIPPARCOS parallax $\pi_{\text {trig }}$, bolometric flux $F_{\mathrm{Bol}}$ ) and observational parameters (observing date, number of obtained scans, names of calibrator stars and their estimated diameters $\Theta_{\text {Cal. }}$ ). Spectral Type, $m_{\mathrm{V}}$, and $\pi_{\text {trig }}$ are taken from the HIPPARCOS catalogue (Perryman \& ESA 1997), references for $F_{\text {Bol }}$ are cited below. Note, HR 7635 was classified as K5-M0 by Morgan \& Keenan (1973), as K5 before.

\begin{tabular}{|c|c|c|c|c|c|c|c|c|c|c|c|}
\hline HR & FK5 & Name & $\begin{array}{r}\text { Spectral } \\
\text { Type }\end{array}$ & $\begin{array}{r}m_{\mathrm{V}} \\
{[\mathrm{mag}]}\end{array}$ & $\begin{array}{r}\pi_{\text {trig }} \\
{[\text { mas }]}\end{array}$ & $\begin{array}{r}F_{\mathrm{Bol}} \\
{\left[10^{-17} \mathrm{~W} / \mathrm{m}^{2}\right]}\end{array}$ & Ref. & $\begin{array}{l}\text { Observing } \\
\text { Dates }\end{array}$ & $\begin{array}{l}\text { \# of } \\
\text { scans }\end{array}$ & $\begin{array}{l}\text { Calibr. } \\
\text { stars }\end{array}$ & $\begin{array}{l}\Theta_{\text {Cal. }} \\
{[\mathrm{mas}]}\end{array}$ \\
\hline 5299 & 1368 & BY Boo & $\mathrm{M} 4.2 \mathrm{III}$ & 5.13 & $7.01 \pm 0.66$ & $2.52 \pm 0.40$ & $\mathrm{a}$ & $\begin{array}{l}2000-07-07 \\
2000-07-13\end{array}$ & $\begin{array}{l}2 \\
4\end{array}$ & FK5 527 & 0.5 \\
\hline 7635 & 752 & $\gamma$ Sge & K5III & 3.51 & $11.90 \pm 0.71$ & $2.792 \pm 0.14$ & $\mathrm{~b}$ & 2000-07-21 & 6 & FK5 768 & 0.3 \\
\hline 8621 & & V416 Lac & M4III & 5.11 & $7.59 \pm 0.57$ & & & $\begin{array}{l}2000-07-07 \\
2000-07-12 \\
2000-07-13\end{array}$ & $\begin{array}{l}2 \\
2 \\
3\end{array}$ & $\begin{array}{l}\text { FK5 } 891 \\
\text { HR } 8494\end{array}$ & $\begin{array}{l}0.3 \\
0.8\end{array}$ \\
\hline
\end{tabular}

References for bolometric fluxes: (a) Tsuji (1981), (b) Alonso et al. (1999).
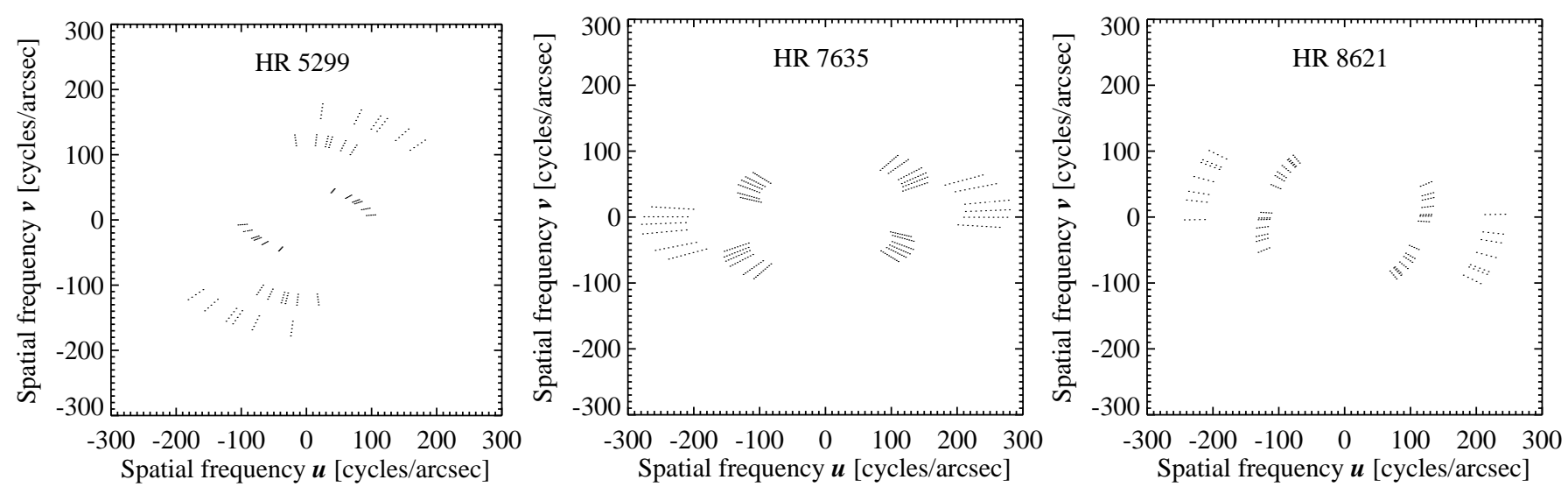

Fig. 1. Plot of the obtained coverages of the $u v$-plane of the HR 5299, HR 7635 and HR 8621 observations based on all observation dates (see Table 1) and on those spectral channels which were used for the data analysis, i.e. the 10 reddest channels for HR 7635 and the 5 reddest channels for the fainter stars HR 5299 and HR 8621.

spectral channel by Fourier transform of the bin counts as a function of time, (2) calculation of the squared visibility amplitudes $\left(\left|V_{i}\right|^{2}\right)$ and amplitude $\left(\left|V_{123}\right|\right)$ and phase $\left(\phi_{123}\right.$, "closure phase") of the complex triple product, averaged over the $1 \mathrm{~s}$ intervals, (3) editing of the $1 \mathrm{~s}$ data, (4) further averaging over a scan of $90 \mathrm{~s}$, and (5) calibration of the program star's squared visibility amplitudes, triple amplitudes, and closure phases by normalization with the corresponding smoothed (time kernel of $20 \mathrm{~min}$ ) quantities of the calibrator stars.

In step (2) $|V|^{2}$ is calculated using the unbiased estimator

$|V|^{2}=\underbrace{4\left[\frac{\pi / n}{\sin (\pi / n)}\right]^{2}}_{=: f} \frac{<X^{2}+Y^{2}-\sigma_{I}^{2}(N)>}{<N>^{2}}$,

where $n=8$ is the number of bins, $N$ the total photon count rate in $2 \mathrm{~ms}$, and $\sigma_{I}^{2}$ the variance of the intensity caused by photon and detection noise. For stellar observations, $|V|^{2}$ is compensated for background intensity by an additional factor $\left.\langle N\rangle^{2} /<N-D\right\rangle^{2}$, where $D$ is the background rate. The use of a squared quantity requires attention to the bias term $\sigma_{I}^{2}$ which is discussed in detail below. The triple product in step (2) is calculated using the unbiased estimator

$\left|V_{123}\right| \mathrm{e}^{i \phi_{123}}=<\left(X_{1}+i Y_{1}\right)\left(X_{2}+i Y_{2}\right)\left(X_{3}+i Y_{3}\right)>$.

The phase $\phi_{123}$ of the complex triple product, formed by a triangle of baselines, is not corrupted by the phase noise caused by atmospheric turbulence (Jennison 1958). No bias correction is required for the triple product, since the noise from the three detector arrays receiving the signal from each baseline is uncorrelated (e.g. Hummel et al. 1998). In a triple with two short baselines and one long baseline resolving a stellar disk, as in the configuration used here, the triple amplitude has a higher signal-tonoise ratio than the squared visibility amplitude on the long baseline. This effect can be seen in our data shown below. It can be understood due to the fact that the low visibility on the long baseline is not squared but multiplied with higher amplitudes from the other two baselines in the triple. This effect was also confirmed by simulations of visibility data based on Poisson noise. For the long baseline a visibility amplitude of 0.1 was assumed and for the two short baselines a visibility amplitude of 0.6. 
The signal-to-noise ratio of the squared visibility on the long (east-west) baseline was found to be 5 while that of the triple product was 20 .

Formal errors for the squared visibility amplitudes, triple amplitudes and closure phases were calculated based on the scatter of the $1 \mathrm{~s}$ samples. Calibration errors of the squared visibility amplitudes were estimated to be $7 \%$ for HR 7635 and 10\% for HR 5299 and HR 8621 based on comparisons of different scans and on calibrations with other calibrator stars at slightly farther distances in time and position. The total (formal and calibration) errors for the triple amplitudes and closure phases of HR 7635, HR 5299, and HR 8621 were estimated to be 2, 1.5, and 2 times the formal errors, respectively.

Noise bias compensation. The use of the squared quantity $|V|^{2}$ requires attention to bias corrections as shown by Hummel et al. (1998) for NPOI data, by Colavita (1999) for the case of the Palomar Testbed Interferometer, and by Davis et al. (1999) for the Sydney University Stellar Interferometer. The noise bias term $\sigma_{I}^{2}$ in Eq. (1) is equal to $N$ in case of Poisson statistics (e.g. Shao et al. 1988). Since the NPOI detectors exhibit non-Poisson noise, due to after-pulsing of the APDs, the noise bias is estimated by $\sigma_{I}^{2}=Z^{2}$ (Hummel et al. 1998). Here, $Z^{2}$ is the fringe amplitude floor estimated at four times the modulation frequency ( $X$ and $Y$ are calculated at the temporal frequency $k=1$ to select the component corresponding to the modulation frequency of the delay lines). However, despite the $Z^{2}$ bias compensation, positive visibility amplitudes are observed for fringeless data and this bias, $B:=|V|^{2}$ ( fringeless data, $\sigma_{I}^{2}=Z^{2}, D=0$ ), was found to be a function of $N$ and to be different for each spectral channel $c$ and each spectrometer $i$. It was modeled with a power-law

$B(i, c)=a_{0}(i, c) N^{a_{1}(i, c)}$

where parameters $a_{0}$ and $a_{1}$ were fitted to the fringeless data. This additional bias was compensated by using

$\sigma_{I}^{2}(N)=Z^{2}+B N^{2} / f$,

in Eq. (1). Obtained ranges for parameters $a_{0}$ and $a_{1}$ are $[0.25,3.4]$ and $[-1.48,-0.93]$, respectively. Thus, $B$ is approximately inversely proportional to $N$, i.e. the star's brightness. Consequently, its magnitude relative to the squared visibility amplitude is largest for low visibility values of faint stars. Therefore, the compensation of this additional noise bias is essential to obtain the very low squared visibility amplitudes of our faint program stars around and beyond the first minimum with a precision that allows an analysis of the limb-darkened intensity profiles. For instance, for our stars HR 5299, HR 7635, and HR 8621, the total biases $\sigma_{I}^{2} f / N^{2}$ for the bluest used channel and the $37.5 \mathrm{~m}$ baseline amount to about 0.056, 0.063, and 0.063, respectively. Residuals of $\sim 0.004, \sim 0.004$, and $\sim 0.003$ remain after the $Z^{2}$ correction and are compensated by $B$. For comparison, the squared visibility amplitude of a fully darkened disk at the second maximum has a value of 0.0074 .

The triple amplitudes and closure phases are not affected by this bias, as mentioned above.

\section{Data analysis and results}

Figures 2, 3 and 4 show all obtained squared visibility amplitude, triple amplitude, and closure phase data of HR 5299, HR 7635, and HR 8621, respectively. Also shown are best fitting uniform disk models, fully darkened disk models, and Kurucz stellar model atmospheres as described below.

The measured squared visibility amplitude of all three program stars as a function of increasing $u v$-radius decreases monotonously towards a minimum, beyond which they increase. The low values are difficult to measure since they correspond to vanishing fringe contrasts. The measurement of almost zero squared visibility amplitude values with acceptable error bars at the minima confirms the feasibility of the bootstrapping technique as well as the correctness of the photon and detection noise bias compensation. The observed functional form of the squared visibility amplitude is expected for a disklike object intensity distribution. The consistency of visibility values with baselines of different orientations excludes large deviations from circular symmetry. The absence of systematic variations with smaller spatial frequencies excludes additional large-scale structures like circumstellar material with considerable intensity.

The triple amplitudes show a similar behaviour for decreasing wavelength, i.e. increasing spatial resolution, with a minimum at a wavelength where the closure phases clearly exhibit a flip from 0 to $\pi$. The absence of intermediate closure phases indicates object intensity distributions which are symmetric through reflection. Thus, for spectral channels with a bandwidth covering the location of the phase flip, intermediate values can occur. For HR 8621, all recorded triple amplitudes and closure phases were beyond the minimum and the flip, due to the star's large diameter in relation to the effective baseline lengths. The triple amplitudes and closure phases show a higher signalto-noise ratio than the squared visibility amplitudes on the long baseline, as discussed in Sect. 3 .

The complex visibility of an astronomical object is related to the object intensity distribution through a Fourier transform. Consequently, the object intensity distribution can in principle be directly reconstructed from interferometric data using imaging techniques which effectively interpolate the limited coverage of the $u v$-plane. This was performed with NPOI data e.g. by Benson et al. (1997) for the double star Mizar A and by Hummel et al. (1998) for Matar. However, in order to obtain accurate estimates of physical parameters, model fits are a better choice. This applies especially to stellar disks since their imaging would require more resolution elements. Here, our data provide, roughly, two resolution elements across the diameter of the stars. The strength of the limb-darkening is related to 

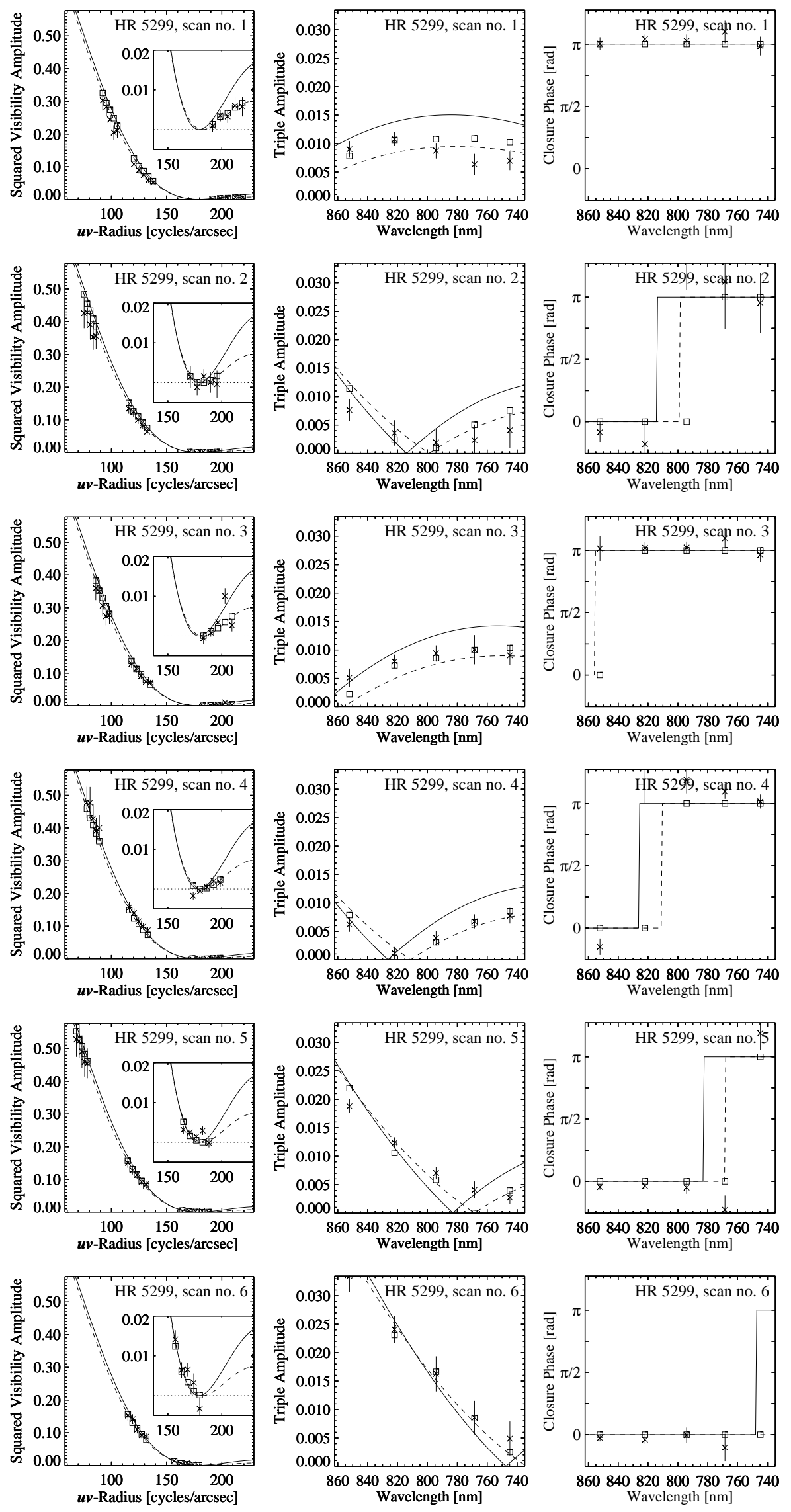

Fig. 2. Squared visibility amplitudes (left), triple amplitudes (middle), and closure phases (right) of HR 5299 and of best fitting models, for all obtained scans. The squared visibility amplitudes are plotted as a function of the $u v$-radius, and the triple amplitudes and closure phases as a function of the spectral channels' central wavelengths. The inset plots (left) provide an enlarged view of the low squared visibility amplitude values around and beyond the first minimum. The $\mathrm{x}$-symbols with error bars indicate the observations and their errors, the solid line the model values based on a uniform disk, the dashed line those based on a fully darkened disk, and the squares those based on the best fitting Kurucz stellar model atmosphere (Kurucz 1993). The error bars include the formal errors as well as the calibration errors as described in Sect. 3. 
986
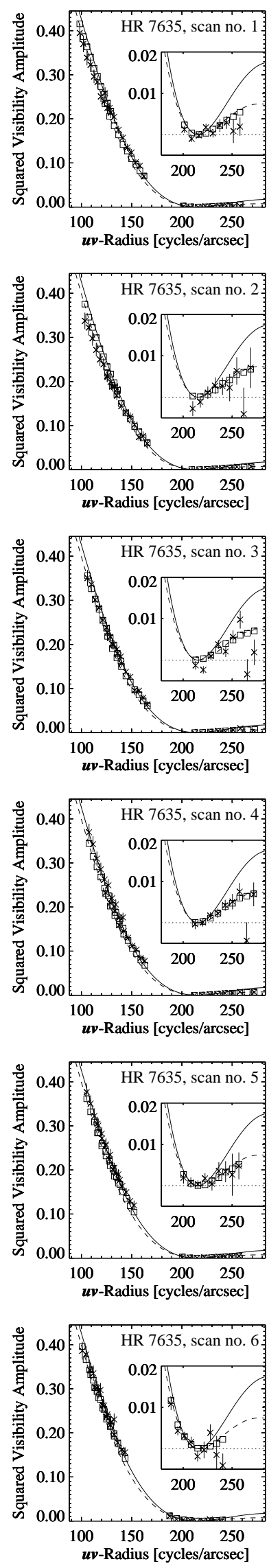

M. Wittkowski et al.: Limb-darkening measurements with NPOI
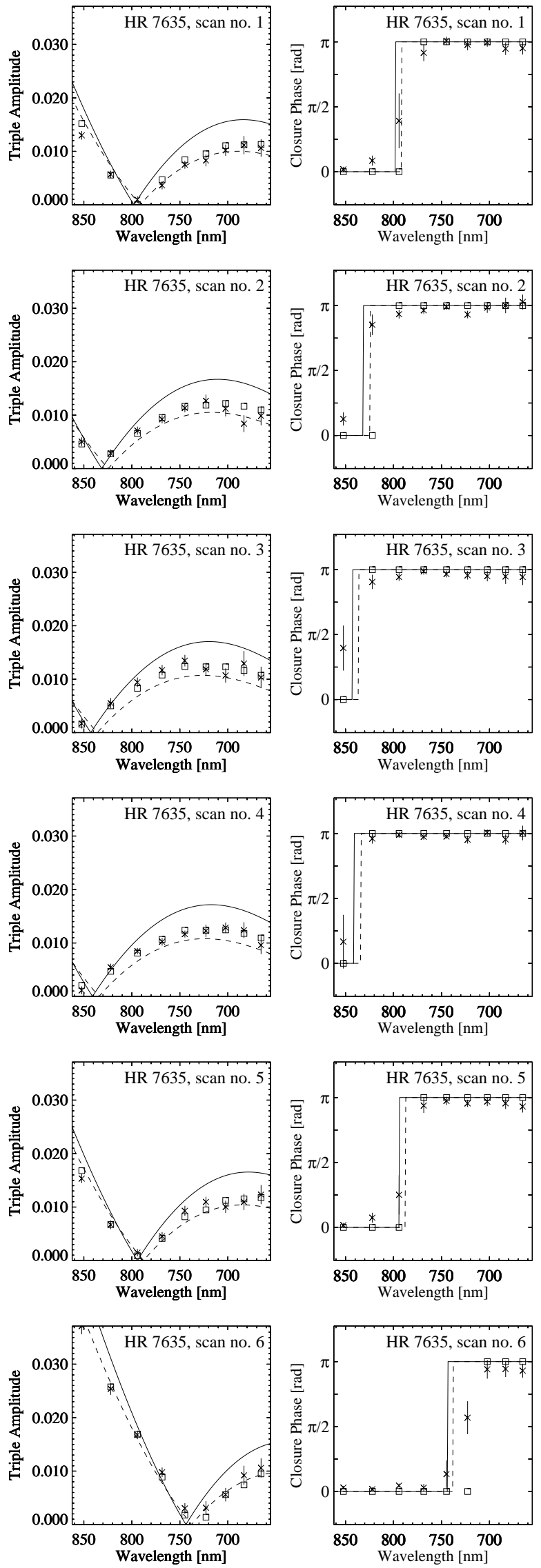

Fig. 3. As Fig. 2, but for HR 7635. 

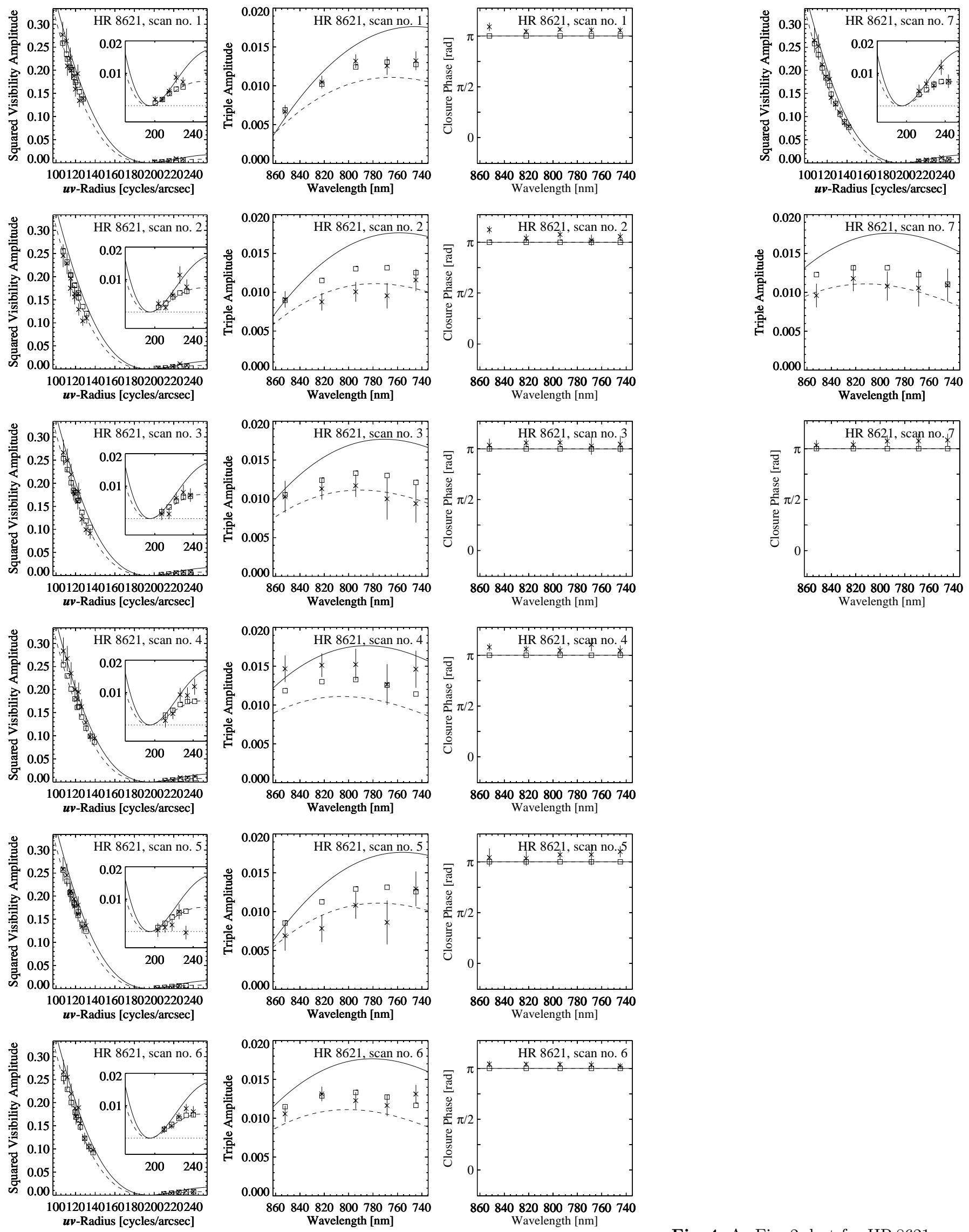

Fig. 4. As Fig. 2, but for HR 8621. 
Table 2. Best fitting diameters of HR 5299, HR 7635, and HR 8621 based on the models of a uniform disk (UD), a fully darkened disk (FDD) and the best fitting Kurucz model atmosphere (best K.) together with the obtained $\chi_{\nu}^{2}$ values. For HR 5299, HR 7635, and HR 8621 the numbers of degrees of freedom are 150,300 , and 175 , respectively. The quantity $\epsilon_{\text {final }}$ denotes the final error of the diameter based on the best Kurucz model atmosphere due to the formal error $\left(\epsilon_{\text {formal }}\right)$ and calibration error $\left(\epsilon_{\text {calibr. }}\right)$ of the underlying data as well as on an error resulting from the choice of $T_{\text {eff }}$ and $\log g\left(\epsilon_{\text {model }}\right)$. The last four rows give the mean values and standard deviations of the UD and FDD diameters determined for each spectral channel separately and corrected with factors derived from the expected model atmosphere.

\begin{tabular}{l|ll|ll|ll}
\hline & \multicolumn{2}{|c|}{ HR 5299 } & \multicolumn{2}{c|}{ HR 7635 } & \multicolumn{2}{c}{ HR 8621 } \\
& \multirow{2}{|c|}{$\Theta$} & $\chi_{\nu}^{2}$ & $\Theta$ & $\chi_{\nu}^{2}$ & $\Theta$ & $\chi_{\nu}^{2}$ \\
& {$[\mathrm{mas}]$} & & {$[\mathrm{mas}]$} & & {$[\mathrm{mas}]$} & \\
\hline $\mathrm{UD}$ & 6.82 & 3.15 & 5.67 & 4.36 & 6.25 & 4.23 \\
FDD & 7.85 & 1.38 & 6.61 & 2.26 & 7.39 & 1.67 \\
best K. & 7.44 & 1.15 & 6.18 & 1.17 & 6.94 & 1.31 \\
$\epsilon_{\text {formal }}$ & 0.03 & & 0.01 & & 0.03 & \\
$\epsilon_{\text {calibr. }}$ & 0.10 & & 0.07 & & 0.10 & \\
$\epsilon_{\text {model }}$ & 0.02 & & 0.02 & & 0.05 & \\
$\epsilon_{\text {final }}$ & 0.11 & & 0.07 & & 0.12 & \\
corr. $d_{\text {UD }}$ & 7.45 & & 6.11 & & 6.92 & \\
$\sigma\left(d_{\text {UD }}\right)$ & 0.07 & & 0.02 & & 0.08 & \\
corr. $d_{\text {FDD }}$ & 7.50 & & 6.13 & & 6.95 & \\
$\sigma\left(d_{\text {FDD }}\right)$ & 0.06 & & 0.02 & & 0.08 & \\
\hline
\end{tabular}

the height of the second maximum of the visibility function. The diameter is then determined by the locations of the minima of the visibility function and of the flip of the closure phases.

Uniform disk and fully darkened disk models. Since the data as described above suggest a circularly symmetric disklike object intensity distribution $I$, models of a uniform disk (UD, $I=1$ for $0 \leq \mu \leq 1, I=0$ otherwise, $\mu=\cos \Theta$ being the cosine of the angle between the line of sight and the normal of the surface element of the star) and a fully darkened disk (FDD, $I=\mu$ ) are used as a first approach to describe the data. In these cases, the visibility functions are given by

$V_{\mathrm{UD}}=\frac{2 J_{1}\left(x_{\mathrm{UD}}\right)}{x_{\mathrm{UD}}} \quad V_{\mathrm{FDD}}=\frac{3 \sqrt{\pi} J_{3 / 2}\left(x_{\mathrm{FDD}}\right)}{\sqrt{2} x_{\mathrm{FDD}}^{3 / 2}}$

with $x_{\mathrm{UD}}$, FDD $=\pi \Theta_{\mathrm{UD}, \mathrm{FDD}} \sqrt{u^{2}+v^{2}}$ a dimensionless spatial frequency $(u, v$ : spatial frequencies [cycles/arcsec] as in Figs. 1-4, $\Theta$ : angular diameter of the star) and $J_{1}$ and $J_{3 / 2}$ the Bessel function of first kind and orders 1 and $3 / 2$ (see e.g. Hestroffer 1997). With $V$ calculated for each baseline $i \in\{1,2,3\}$, the squared visibility amplitudes $\left|V_{i}\right|^{2}$, the amplitude of the triple amplitude $\left|V_{1} V_{2} V_{3}\right|$ and the closure phase $\phi_{1}+\phi_{2}-\phi_{3}\left(\phi_{i}=0\right.$ where $V_{i}>0$ and $\phi_{i}=\pi$ where $\left.V_{i}<0\right)$ can be derived.

A $\chi^{2}$ minimization algorithm (simplex method) was applied in order to find the best fitting angular diameters
$\Theta_{\text {UD }}$ and $\Theta_{\mathrm{FDD}}$, using all available data, i.e. the squared visibility amplitudes, the triple amplitudes and the closure phases. The derived diameters are shown in the first two rows of Table 2, together with the corresponding reduced $\chi_{\nu}^{2}$ values. For HR 5299, HR 7635, and HR 8621 the numbers of degrees of freedom are 150,300 , and 175 , respectively. These $\chi_{\nu}^{2}$ values show that the UD model can be rejected and that the FDD model is a better description of our data. The model functions are indicated by solid lines (UD) and dashed lines (FDD) in Figs. 2-4. The minima of the visibility and triple amplitude functions are well defined by the data. Before and in particular beyond the minima, our measured triple amplitudes for all three stars are significantly lower than the UD model values and slightly higher than the FDD model values. This indicates limb-darkened disks, less extreme than fully darkened disks, as predicted for late-type giants. Therefore, the specific limb-darkened profiles were investigated as described in the following paragraphs.

Uniform disk and fully darkened disk models corrected for the effect of limb-darkening. Before we present a direct fit of all our data to theoretical model atmospheres, we take an alternative approach that is based on results which are independent of a particular model atmosphere.

Model atmospheres predict a decrease of the degree of limb-darkening with increasing wavelength, i.e. a transition from near-FDD to near-UD continuum shapes, while the "true" limb-darkened diameter is wavelengthindependent (Manduca et al. 1977; Hofmann \& Scholz 1998). Since our data provide information at different spectral channels, they can be used to test stellar model atmospheres on their predicted wavelength dependence of UD and FDD diameters.

Recently, Hestroffer (1997) discussed a limb-darkening law $I_{\lambda}=\mu^{\alpha}$, with $\alpha$ a positive real number, as empirical brightness distribution function. This representation of the center-to-limb variation which uses only one limbdarkening coefficient includes the UD and FDD models and is very well suited to describe a wide range of different realistic limb-darkening shapes (Hofmann \& Scholz 1998). Thus, in principle our data could be used to simultaneously determine both the apparent limb-darkened diameter of our program stars and their limb-darkening parameter $\alpha_{\lambda}$ for each spectral channel. However, by means of Monte-Carlo simulations based on the actual coverage of the $u v$-plane of these observations and the claimed precision, it was found that this determination is ambiguous.

The employed method was first used by Hanbury Brown (1974), then applied by Quirrenbach et al. (1996) and recently used for theoretical studies by Hofmann \& Scholz (1998) and Davis et al. (2000). The diameters based on UD and FDD models were derived for each spectral channel separately using only the squared visibility amplitudes up to the first minimum, since these data can well be described by UD and FDD models. The triple amplitudes and closure phases were not used since most of them 

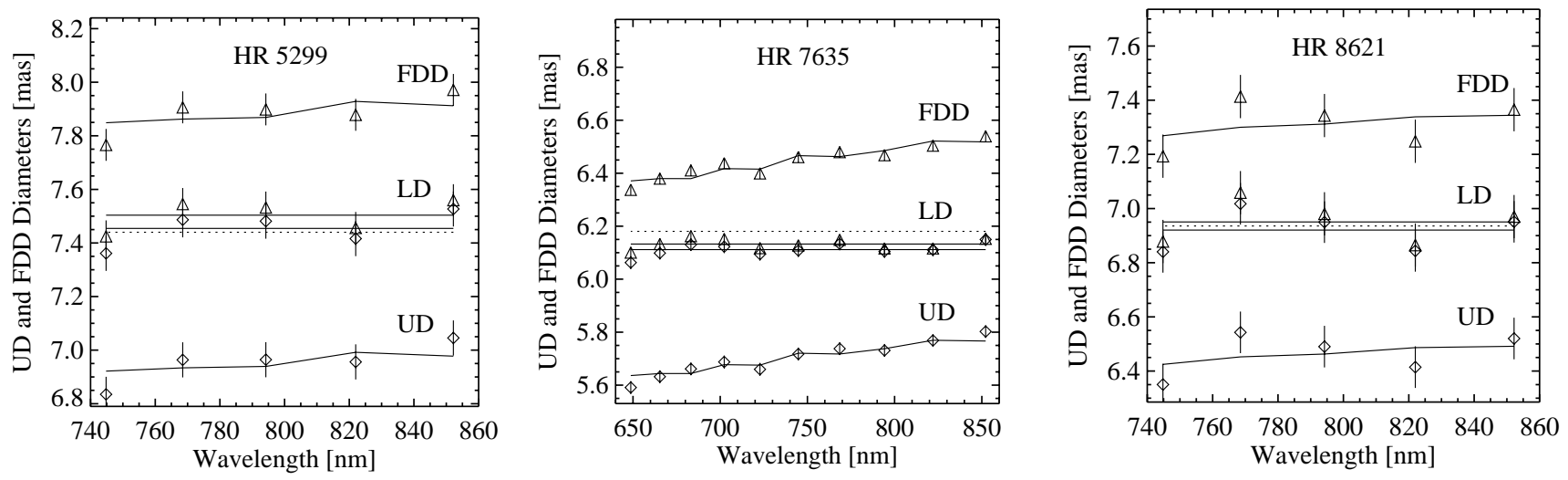

Fig. 5. Diameters based on uniform disk (UD, diamonds) and fully darkened disk (FDD, triangles) models for each spectral channel together with the Kurucz model atmosphere predictions (connected by solid lines). In addition, the limb-darkened (LD) diameters are shown which were derived by applying correction factors to the UD and FDD diameters. The mean LD diameters as quoted in Table 2 are indicated by the solid lines. The error bars represent the standard deviation of the obtained LD diameters. For comparison, the dotted lines indicate the limb-darkened diameters which were derived by the direct fit to a grid of Kurucz model atmospheres.

contain visibility values beyond the first minimum, which do not fit UD and FDD models. The resulting UD and FDD diameters were multiplied by limb-darkening correction factors to obtain the limb-darkened diameter of the star. Following the authors mentioned above, the correction factors were derived as the ratios $x_{\mathrm{LD}, 0.3} / x_{\mathrm{UD}} 0.3$ and $x_{\mathrm{LD}, 0.3} / x_{\mathrm{FDD}, 0.3}$ with $x_{\mathrm{LD}, 0.3}, x_{\mathrm{UD}, 0.3}=2.0818$, and $x_{\mathrm{FDD}, 0.3}=2.3451$ being the spatial frequencies where the squared visibility amplitudes $\left|V_{\mathrm{LD}}\right|^{2},\left|V_{\mathrm{UD}}\right|^{2}$ and $\left|V_{\mathrm{FDD}}^{2}\right|$ (see Eqs. (5) and (6)) equal 0.3. For the determination of $x_{\mathrm{LD}}$, the Kurucz model atmosphere as described in detail in the following section was used with values for $T_{\text {eff }}$ and $\log g$ according to the spectral type of the star.

The obtained diameters are plotted in Fig. 5 as a function of wavelength. The mean values and standard deviations of the corrected UD and FDD diameters are shown in the last four rows of Table 2. Our observed wavelength dependence of the UD and FDD diameters corresponds well with the model predictions. The small predicted deviations from a monotonous wavelength dependence are caused by effects of molecular absorption bands. Especially in the case of the brightest of our program stars, HR 7635, our obtained diameters match the model predictions very well. Here, a larger number of spectral channels could be used and the effective temperature causes the transition from the UD to FDD model to occur in the observed wavelength range. For all our program stars, no systematic deviations between observations and model predictions occur; which indicates the correctness of the atmosphere models used. Consequently, the derived limb-darkened diameters are, as required, independent of wavelength. The results based on the UD fit and those based on the FDD fit are consistent.

The uncorrected UD and FDD diameters can be used for future comparisons with other model atmospheres and, furthermore, for future analyses taking additional information at other wavelengths into account. However, this approach cannot make use of our data at long baselines
Table 3. Comparison of minimum $\chi_{\nu}^{2}$ values obtained by fitting our HR 5299 and HR 7635 data to a grid of Kurucz model atmospheres with solar chemical abundances (Kurucz 1993) based on effective temperatures ranging from $3500 \mathrm{~K}$ to $4500 \mathrm{~K}$ and $\log g$ ranging from 0.0 to 2.5. For each program star, the best $\chi_{\nu}^{2}$ values are marked by a box. For HR 5299 and HR 7635 the numbers of degrees of freedom are 150 and 300, respectively.

\begin{tabular}{|c|c|c|c|c|c|c|c|}
\hline HR & $\begin{array}{r}\log g / \\
T_{\text {eff }} \\
{[\mathrm{K}]}\end{array}$ & 0.0 & 0.5 & 1.0 & 1.5 & 2.0 & 2.5 \\
\hline \multirow[t]{5}{*}{5299} & 3500 & 1.25 & 1.19 & 1.15 & 1.17 & 1.19 & 1.23 \\
\hline & 3750 & 1.19 & 1.19 & 1.19 & 1.18 & 1.18 & 1.17 \\
\hline & 4000 & 1.21 & 1.21 & 1.21 & 1.20 & 1.20 & 1.20 \\
\hline & 4250 & 1.27 & 1.27 & 1.26 & 1.26 & 1.26 & 1.26 \\
\hline & 4500 & 1.42 & 1.40 & 1.38 & 1.37 & 1.36 & 1.35 \\
\hline \multirow[t]{5}{*}{7635} & 3500 & 1.50 & 1.40 & 1.35 & 1.38 & 1.47 & 1.58 \\
\hline & 3750 & 1.29 & 1.29 & 1.29 & 1.29 & 1.27 & 1.26 \\
\hline & 4000 & 1.18 & 1.17 & 1.17 & 1.17 & 1.18 & 1.18 \\
\hline & 4250 & 1.20 & 1.20 & 1.19 & 1.19 & 1.19 & 1.19 \\
\hline & 4500 & 1.26 & 1.25 & 1.24 & 1.23 & 1.23 & 1.23 \\
\hline
\end{tabular}

and the corresponding triple amplitudes and closure phases. In order to provide more accurate limb-darkened diameters and to discriminate between different model assumptions a direct fit of all our data to Kurucz model atmospheres is described in the following paragraph.

Comparison of our interferometric data with a grid of Kurucz stellar model atmospheres. In an effort to compare different model atmosphere predictions based on a grid of effective temperatures and surface gravities with our interferometric data, rather than assuming a particular model profile a priori, the best $\chi_{\nu}^{2}$ values based on different models were determined. In this way, theoretically predicted differences of the strength of the limb-darkening effect for 
different effective temperatures and surface gravities can be compared to direct measurements.

Kurucz (1993) tabulates monochromatic intensities $I(1)$ and limb-darkening ratios $I(\mu) / I(1)$ for 17 values of $\mu$ in 1221 frequency intervals ranging from $9.09 \mathrm{~nm}$ to $160.0 \mu \mathrm{m}$, based on grids of model atmospheres for different chemical abundances. Here, his grid for solar chemical abundances and a microturbulent velocity of $v_{\text {turb }}=2 \mathrm{kms}^{-1}$ was used (file "cfaku5.harvard. edu/grids/gridP00/ip00k2.pck19"). The data is available for effective temperatures $T_{\text {eff }}$ ranging from $3500 \mathrm{~K}$ to $50000 \mathrm{~K}$ in steps of $250 \mathrm{~K}$ (for low $\left.T_{\text {eff }}\right)$ and surface gravities $\log g(\mathrm{cgs})$ from 0 to 5 in steps of 0.5 . For all our program stars, $T_{\text {eff }} \leq 4000 \mathrm{~K}$ and $\log g \leq 2$ are predicted, based on their spectral types (see Table 4). Thus, only models with $T_{\text {eff }} \in[3500 \mathrm{~K}, 4500 \mathrm{~K}]$ and $\log g \in[0,2.5]$ were considered.

NPOI passband-specific limb-darkening ratios $I_{\mathrm{c}}(\mu) / I_{\mathrm{c}}(1)$ were calculated by integrating the Kurucz data over each of the NPOI spectral channel's $c \in[1,10]$ sensitivity functions. The NPOI spectral channels may be affected by molecular absorption bands, which is taken into account by the calculation of passband-specific limb-darkening model profiles. Compact photospheres were assumed, i.e. $I_{\mathrm{c}}(\mu=0)=0 . I_{\mathrm{c}}(1)$ was set to 1 since our measured visibility values are scaled to $V(0)=1$ for each spectral channel separately.

Following Davis et al. (2000), the model visibility values were derived by numerical evaluation of the Hankel transform of the obtained tabulated intensity profiles

$V_{\mathrm{LD}}=\frac{\int_{0}^{1} I_{\mathrm{c}}(\mu) J_{0}\left[x_{\mathrm{LD}}\left(1-\mu^{2}\right)^{1 / 2}\right] \mu \mathrm{d} \mu}{\int_{0}^{1} I_{\mathrm{c}}(\mu) \mu \mathrm{d} \mu}$,

with $x_{\mathrm{LD}}=\pi \Theta_{\mathrm{LD}} \sqrt{u^{2}+v^{2}}$ the dimensionless spatial frequency as described above, but now based on the star's limb-darkened angular diameter $\Theta_{\mathrm{LD}}$. No approximation of the tabulated model limb-darkened profiles by any limbdarkening law was used. The model squared visibility amplitudes, model triple amplitudes, and model closure phases were derived as described above.

Based on each of the 30 considered Kurucz models (five values for $T_{\text {eff }}$ and six values for $\log g$ ), the wavelengthindependent limb-darkened diameter, treated as the only free parameter, and the corresponding $\chi_{\nu}^{2}$ value were derived as described above using all our available data, i.e. the squared visibility amplitudes, the triple amplitudes, and the closure phases.

For our three program stars, the lowest obtained $\chi_{\nu}^{2}$ values are listed in Table 2, together with the obtained best fitting limb-darkened diameters. Table 3 shows all resulting $\chi_{\nu}^{2}$ values for HR 5299 and HR 8621, where the lowest $\chi_{\nu}^{2}$ values are marked by a box.

The occurrence of minimum $\chi_{\nu}^{2}$ values larger than 1.0 might, in principle, be caused by optimum parameters lying in between our grid points, a wrong model assumption, an underestimation of the calibration errors, or systematic calibration errors. An underestimation of the calibration errors leading to total errors underestimated by only $7 \%$ and $8 \%$ is most likely to be the main cause for the deviations from unity in the cases of HR 5299 and HR 7635 since these errors can only be roughly estimated (see Sect. 3). A considerable part of the larger deviation from unity, a value of 1.31 , in the case of HR 8621 might also be caused by an effective temperature lower than $3500 \mathrm{~K}$, i.e. an incorrect model assumption, or systematic effects during the calibration process. The stellar atmosphere model was adopted as the best fit to our data for HR 5299 and HR 7635. The $\chi_{\nu}^{2}$ values were analyzed as a function of $T_{\text {eff }}$ and $\log g$ for these program stars only, as follows.

For these program stars, significantly different $\chi_{\nu}^{2}$ values are obtained for different model parameters, for example, in the case of HR 5299 we obtain values between 1.15 and 1.42. At higher temperatures, differences for varying $T_{\text {eff }}$ are larger than for varying $\log g$, because of the latter's lesser effect on the limb-darkened profile (see e.g. Manduca et al. 1977).

To take the deviations of the $\chi_{\nu}^{2}$ values from unity into account, the values in Table 3 were normalized to unity at the minimum for the following analysis. Using this method, the total assumed errors in our data are increased by common mean factors of 1.07 and 1.08 , neglecting that calibration errors depend on the value of the visibility. It was verified that due to the smallness of this correction the results obtained are still valid and that it is insignificant whether the total data errors are rescaled or just the calibration errors.

Near the minimum, the $\chi^{2}$ function is expected to be a quadratic function of each of the varied parameters. Therefore, for each star, a parabola was fitted to the $\chi^{2}$ values as a function of $T_{\text {eff }}$ with fixed best-fitting $\log g$ and as a function of $\log g$ with fixed best-fitting $T_{\text {eff }}$. Here, more digits were used than shown in Table 3 . The most likely values for the parameters $T_{\text {eff }}$ and $\log g$ can be estimated by the locations of the minima of the parabola. Assuming purely Gaussian noise, the corresponding $1 \sigma$ errors can be estimated as the variation in the parameters which will increase the normalized total $\chi^{2}$ values by 1 from its value at the minimum of the fitted parabola (see e.g. Bevington \& Robinson 1991). For HR 5299 and HR 7635, the $\chi^{2}$ values as a function of $T_{\text {eff }}$ as well as of $\log g$ match a parabola very well. This confirms that effects due to systematic calibration errors or an incorrect model assumption are not of considerable size. However, small additional errors due to these effects cannot be ruled out and are not included in the error analysis presented here. For HR 5299, the $\chi_{\nu}^{2}$ values in Table 3 as a function of $T_{\text {eff }}$ extend to the minimum but not beyond. However, the one-sided $\chi_{\nu}^{2}$ values fit a parabola with a minimum at $3520 \mathrm{~K}$ very well, confirming that our grid point at $3500 \mathrm{~K}$ is in fact close to the minimum. The derived most likely values and the errors are shown in Table 4 and are compared to independent estimates in Sect. 5.

For all our program stars, the best $\chi_{\nu}^{2}$ values derived here are significantly better than those based on the FDD model (see Table 2). This effect is most noticeable in the 
case of HR 7635, due to the higher signal-to-noise ratio of the data and the higher $T_{\text {eff }}$ resulting in limb-darkening that is not as close to the FDD case as for the cooler stars.

The limb-darkened diameters corresponding to the best fitting models are shown in Table 2 . Errors $\epsilon_{\text {final }}$ were derived based on the formal errors and calibration errors mentioned in Sect. 3 and on those due to the choice of $T_{\text {eff }}$ and $\log g$. For the latter error, the standard deviations of diameters based on models with $T_{\text {eff }}$ and $\log g$ values within their error bars were taken. For HR 8621 all 30 considered models were included.

The squared model visibility amplitudes, model triple amplitudes, and model closure phases obtained with the best fitting Kurucz model atmosphere are indicated by the squares in Figs. 2-4. They coincide well with our measured data and describe them considerably better than the UD and FDD models. The observed data of HR 8621 differ slightly from the model values which might be explained by calibration errors or a wrong model assumption as mentioned above. The absence of further systematic deviations between the model predictions and our data confirms that extended photospheres with $I(\mu=0)>0$ need not to be considered and that the width of the NPOI spectral channels does not noticeably affect our analysis.

Deviations from featureless symmetric disks. Although, as discussed above, our data indicate a featureless symmetric disk, a formal search for solutions with an asymmetric object intensity distribution was performed in order to validate this interpretation. All our data were fitted to a limb-darkened profile as derived before but with an elliptical shape. Starting values for the position angle of the major axis were chosen from $0^{\circ}$ to $180^{\circ}$ in steps of $10^{\circ}$ and for the axis ratios (major axis/minor axis) two starting values of 1.05 and 1.1 were considered. For the stars HR 5299, HR 7635, and HR 8621, slightly better $\chi_{\nu}^{2}$ values than for the symmetric intensity profile were derived with axes ratios of $1.02,1.01$, and 1.01 , respectively. These small deviations from circular symmetry are not significant for an asymmetric object intensity distribution, but can be caused, for instance, by systematic calibration errors. However, this study does exclude elliptical object intensity distributions with larger axis ratios.

In order to estimate whether additional hot spots, as they were found on the surfaces of $\alpha$ Ori, $\alpha$ Sco, and $\alpha$ Her (see Sect. 1) could be detected in our data, model squared visibility amplitudes, triple amplitudes, and closure phases were calculated for one example based on our best fitting limb-darkening model with one additional hot spot. The spot's intensity was chosen to be only $2 \%$ of the star's intensity, which is clearly less than that of the spots on $\alpha$ Ori, $\alpha$ Sco, and $\alpha$ Her. The spots were assumed to be unresolved and to have a separation of half of the star's limb-darkened radius. Two position angles were considered, namely that of the preferred direction of the $u v$-plane coverage and that perpendicular to it. Figure 6 shows the model predictions, indicating that the existence of such a spot would significantly affect the closure phases and the triple and visibility amplitudes around their minima. The different triple and visibility amplitudes might be modeled by another stellar diameter and limb-darkening profile, but the occurrence of closure phases significantly different from values of 0 and $\pi$, however, could only be explained by an asymmetric intensity distribution. Our data is not consistent with such a noticeable asymmetry. Consequently, it can be concluded that the existence of an unresolved single spot on the surfaces of our program stars with an intensity at least as high as studied above is highly unlikely. A resolved spot is unlikely, too, since its intensity contribution would be higher. However, by an analysis of the closure phases we cannot rule out a centered spot.

\section{Discussion}

Based on the data analysis described in Sect. 4 it follows that all our interferometric data are consistent with featureless circularly symmetric limb-darkened disks. The method of comparing all our interferometric data to a grid of Kurucz model atmospheres allows the determination of the effective temperature and surface gravity without additional information in the cases of HR 5299 and HR 7635 and provides an accurate estimate of the limb-darkened diameter $\Theta_{\mathrm{LD}}$.

Independent estimates for $T_{\text {eff }}$ and $\log g$ can be obtained by empirical calibrations of the spectral type. Additionally, $T_{\text {eff }}$ and the linear limb-darkened diameter $D$ can be derived by using the bolometric flux $F_{\text {Bol }}$ (see Table 1) and the HIPPARCOS parallax $\pi_{\text {trig }}$, together with our value for $\Theta_{\mathrm{LD}}$. The errors are dominated by the uncertainties of $F_{\mathrm{Bol}}$ and $\pi_{\text {trig. }}$. As a result, variations of $\Theta_{\mathrm{LD}}$ have little impact on $T_{\mathrm{eff}}$ and this estimate of $T_{\mathrm{eff}}$ can be regarded as sufficiently independent of our determination by the direct fit to Kurucz model atmospheres as well.

Table 4 lists the results obtained by the direct fit to Kurucz model atmospheres together with the independent estimates. The error estimates for $T_{\text {eff }}$ and $\log g$ are based on the analysis of Table 3 as described above.

Figure 7 compares the two derived $T_{\text {eff values, obtained }}$ by the direct fit to a grid of Kurucz model atmospheres, and by $\Theta_{\mathrm{LD}}$ and $F_{\mathrm{bol}}$, with different empirical calibrations. For HR 8621 the data quality is, as mentioned above, not sufficiently high to obtain $T_{\text {eff }}$ and $\log g$ by means of the direct fit to Kurucz model atmospheres. For HR 5299, both the $T_{\text {eff }}$ value and $\log g$ value derived by this fit to Kurucz model atmospheres are well consistent with the independent estimates. For HR 7635, the obtained $T_{\text {eff }}$ value is higher than that of the independent estimates and consistent only within $\sim 2 \sigma$. The value obtained for $\log g$ is consistent with the empirical calibration.

Our derived values for $\Theta_{L D}$ are generally consistent with earlier determinations of uniform disk diameters corrected for limb-darkening. They are available for HR 5299 (7.0 mas \pm 0.3 mas by Dyck et al. 1996, 1998) 

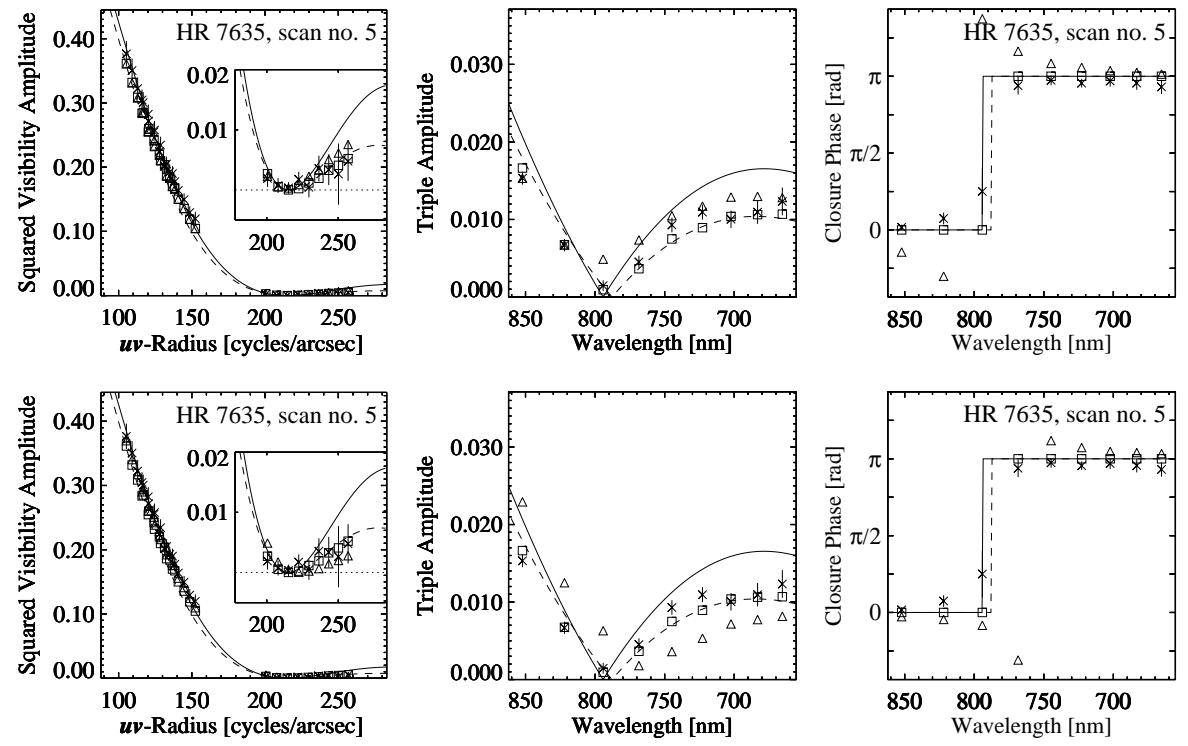

Fig. 6. Impact of hot spots on model data for the example of HR 7635, scan No. 5. The triangles denote the best fitting limb-darkening model with one additional hot spot. The spot's intensity relative to that of the star is $2 \%$, its separation is $\theta_{\mathrm{LD}} / 4$, its position angle is (top) along the preferred direction of the $u v$ plane coverage $\left(3^{\circ}\right)$ and (bottom) perpendicular to it $\left(93^{\circ}\right)$. For comparison, the $\mathrm{x}$-symbols with error bars and the squares denote the measurements and the best fitting limbdarkening model, respectively, as in Fig. 3.

Table 4. Best fitting limb-darkened diameters $\Theta_{\mathrm{LD}}$, effective temperatures, and surface gravities as derived by the direct fit of our interferometric data to a grid of Kurucz model atmospheres as described above (Tables 2 and 3 ). Using $\Theta_{\text {LD }}$ together with the HIPPARCOS parallax $\pi_{\text {trig }}$ and the bolometric flux $F_{\text {Bol }}$ (see Table 1$)$ the linear stellar diameter $D\left(\Theta_{\mathrm{LD}}, \pi_{\text {trig }}\right)$ and the effective temperature $T_{\text {eff }}\left(\Theta_{\mathrm{LD}}, F_{\mathrm{Bol}}\right)$ are derived. For further comparison, the effective temperatures $T_{\mathrm{eff}}(\mathrm{Sp} . \mathrm{T}$.) and surface gravities $\log g$ (Sp.T.) derived from empirical calibrations of the spectral type (Schmidt-Kaler 1982) are shown.

\begin{tabular}{l|lll|ll|ll}
\hline HR & $\begin{array}{l}\Theta_{\mathrm{LD}} \\
{[\mathrm{mas}]}\end{array}$ & $T_{\text {eff }}[\mathrm{K}]$ & $\log g$ & $\begin{array}{l}D\left(\Theta_{\mathrm{LD}}, \pi_{\text {trig }}\right) \\
{\left[R_{\odot}\right]}\end{array}$ & $\begin{array}{l}T_{\text {eff }}\left(\Theta_{\mathrm{LD}}, F_{\mathrm{Bol}}\right) \\
{[\mathrm{K}]}\end{array}$ & $\begin{array}{l}T_{\text {eff }}(\operatorname{Sp} . \mathrm{T} .) \\
{[\mathrm{K}]}\end{array}$ & $\log g(\operatorname{Sp} . \mathrm{T})$. \\
\hline 5299 & $7.44 \pm 0.11$ & $3520 \pm 190$ & $1.3 \pm 0.4$ & $228_{-23}^{+28}$ & $3420 \pm 160$ & 3410 & $<1.3$ \\
7635 & $6.18 \pm 0.07$ & $4160 \pm 100$ & $0.9 \pm 1.0$ & $112_{-8}^{+8}$ & $3850 \pm 70$ & 3950 & 1.7 \\
8621 & $6.94 \pm 0.12$ & - & - & $197_{-17}^{+19}$ & & 3430 & $<1.3$ \\
\hline
\end{tabular}

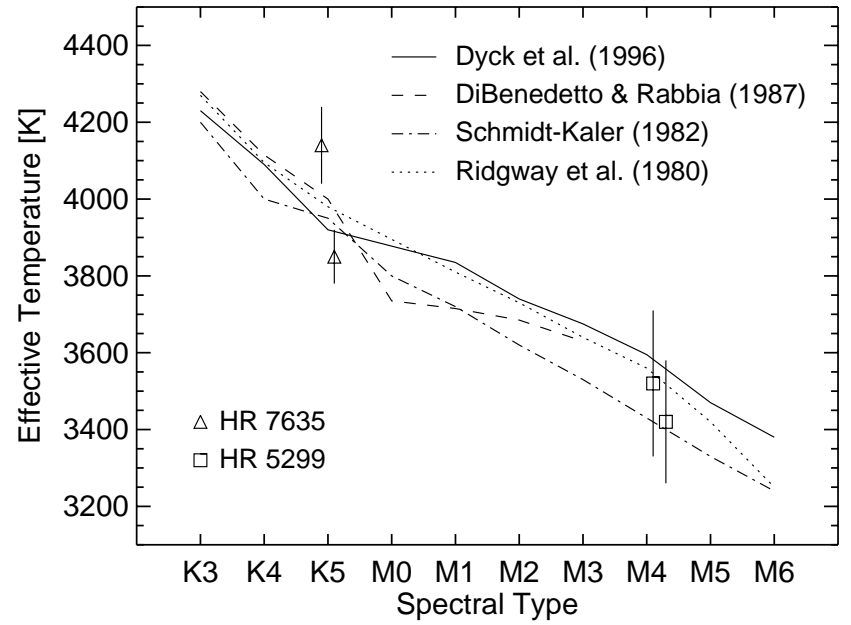

Fig. 7. Comparison of our derived HR 5299 and HR $7635 T_{\text {eff }}$ values with different effective temperature scales (Ridgway et al. 1980; Schmidt-Kaler 1982; DiBenedetto \& Rabbia 1987; Dyck et al. 1996). The symbols shifted to the left of the nominal spectral type position indicate the values derived by the direct fit to a grid of Kurucz model atmospheres. The symbols shifted to the right indicate the values derived from the limb-darkened angular diameter $\Theta_{\mathrm{LD}}$ and the bolometric flux $F_{\text {bol }}$. and for HR 7635 (7.4 mas \pm 0.2 mas by Hutter et al. 1989 ; 5.5 mas \pm 0.5 mas by Dyck et al. 1996, 1998). For HR 7635, Alonso et al. 2000 derived a limb-darkened diameter of 6.12 mas \pm 0.2 mas by means of the infrared flux method (IRFM), wich is in very good agreement with our value.

Our derived linear limb-darkened radii for the three stars of $114 R_{\odot} \pm 13 R_{\odot}, 56 R_{\odot} \pm 4 R_{\odot}$, and $98 R_{\odot} \pm 9 R_{\odot}$ are in good agreement with those obtained with the empirical calibration for M giants by Dumm \& Schild (1998) based on the HIPPARCOS parallaxes, $V$ magnitudes, and $V-I$ color indexes, which are $119 R_{\odot}, 60 R_{\odot}$, and $97 R_{\odot}$, respectively.

The circular symmetry of our observed object intensity distributions is expected because at optical wavelengths only the surfaces of the stars themselves are observed rather than additional circumstellar envelopes where asymmetric morphologies were discovered. These asymmetries in the envelopes can be caused by, e.g., rotations so slow that they do not observably affect the star's shape. The absence of additional surface features as observed on the surfaces of the apparently largest supergiants might be explained by the higher surface gravities of our program giant stars. 


\section{Summary}

Featureless symmetric limb-darkened stellar disks provide good fits to our NPOI interferometric data of HR 5299, HR 7635, and HR 8621. We are able to discriminate between model atmospheres with different effective temperatures and surface gravities. We find that our interferometric measurements and stellar model atmosphere predictions by Kurucz (1993) of the limb-darkening effect are consistent. We obtain high-precision (1\%-2\% accuracy) limb-darkened angular disk diameters and derive linear radii and effective temperatures using the HIPPARCOS parallaxes and bolometric fluxes reported in the literature. With reduced noise terms and the upcoming simultaneous combination of six beams at NPOI we will be able to obtain even more precise limb-darkened diameters of a much larger number of stars. Furthermore, interferometric data will soon allow the discrimination between model atmospheres with different effective temperatures, surface gravities and even chemical abundances with higher precision than in this first attempt. Thus, further observational constraints for model atmospheres will become available, in addition to observations of stellar spectra.

Acknowledgements. We thank the observers B. O'Neill and C. Denison for their careful operation of the NPOI array. M. Wittkowski acknowledges support from the Alexander von Humboldt Foundation through a Feodor Lynen Fellowship. We thank Robert L. Kurucz for making his model atmosphere data easily available. The Navy Prototype Optical Interferometer is a joint project of the Naval Research Lab and the US Naval Observatory in cooperation with Lowell Observatory, and is funded by the Office of Naval Research and the Oceanographer of the Navy. This research has made use of the SIMBAD database, operated at CDS, Strasbourg, France.

\section{References}

Alonso, A., Arribas, S., \& Martínez-Roger, C. 1999, A\&AS, 139,335

Alonso, A., Salaris, M., Arribas, S., Martínez-Roger, C., \& Asensio Ramos, A. 2000, A\&A, 355, 1060

Armstrong, J. T., Mozurkewich, D., Rickard, L. J., et al. 1998, ApJ, 496, 550

Benson, J. A., Hutter, D. J., Elias II, N. M., et al. 1997, AJ, 114(3), 1221

Bevington, P. R., \& Robinson, D. K. 1991, Data Reduction and Error Analysis for The Physical Sciences (McGrawHill), ISBN 0079112439

Burns, D., Baldwin, J. E., Boysen, R. C., et al. 1997, MNRAS, 290, L11

Buscher, D. F., Haniff, C. A., Baldwin, J. E., \& Warner, P. J. 1990, MNRAS, 245, 7p

Colavita, M. M. 1999, PASP, 111, 111

Davis, J., Tango, W. J., Booth, A. J., Thorvaldson, E. D., \& Giovannis, J. 1999, MNRAS, 303, 783
Davis, J., Tango, W. J., \& Booth, A. J. 2000, MNRAS, 318, 387

DiBenedetto, G. P., \& Rabbia, Y. 1987, A\&A, 188, 114

Dumm, T., \& Schild, H. 1998, New Astronon., 3, 137

Dyck, H. M., Benson, J. A., van Belle, G. T., \& Ridgway, S. T. 1996, AJ, 111(4), 1705

Dyck, H. M., van Belle, G. T., \& Thompson, R. R. 1998, AJ, 116,981

Perryman, M. A. C., \& ESA 1997, The HIPPARCOS and TYCHO catalogues, ESA SP Ser., 1200, Noordwijk (Netherlands: ESA Publications Division)

Gilliland, R. L., \& Dupree, A. K. 1996, ApJ, 463, L29

Hajian, A. R., Armstrong, J. T., Hummel, C. A., et al. 1998, ApJ, 496, 484

Hanbury Brown, R., Davis, J., Lake, R. J. W., \& Thompson, R. J. 1974, MNRAS, 167, 475

Haniff, C. A., Scholz, M., \& Tuthill, P. G. 1995, MNRAS, 276, 640

Hestroffer, D. 1997, A\&A, 327, 199

Hofmann, K.-H., \& Scholz, M. 1998, A\&A, 335, 637

Hummel, C. A., Mozurkewich, D., Armstrong, J. T., et al. 1998, AJ, 116, 2536

Hutter, D. J., Johnston, K. J., Mozurkewich, D., et al. 1989, AJ, 340, 1103

Jacob, A. P., Bedding, T. R., Robertson, J. G., \& Scholz, M. 2000, MNRAS, 312, 733

Jennison, R. C. 1958, MNRAS, 118, 276

Kurucz, R. 1993, Limbdarkening for $2 \mathrm{~km} / \mathrm{s}$ grid (No. 13): $[+0.0]$ to $[-5.0]$. Kurucz CD-ROM No. 17 (Cambridge, Mass.: Smithsonian Astrophysical Observatory), 1993

Manduca, A., Bell, R. A., \& Gustafsson, B. 1977, A\&A, 61, 809

Morgan, W. W., \& Keenan, P. C. 1973, ARA\&A, 11, 29

Mozurkewich, D., Johnston, K. J., Simon, R. S., et al. 1991, AJ, 101, 2207

Quirrenbach, A., Mozurkewich, D., Buscher, D. F., Hummel, C. A., \& Armstrong, J. T. 1996, A\&A, 312, 160

Ridgway, S. T., Joyce, R. R., White, N. M., \& Wing, R. F. 1980, ApJ, 235, 126

Roddier, F. 1988, in ESO Conf. and Workshop 29, High Resolution Imaging by Interferometry II, ed. F. Merkle (Garching: ESO), 565

Scholz, M., \& Takeda, Y. 1987, A\&A, 186, 200

Schmidt-Kaler, T. 1982, in Landolt-Börnstein, New Series VI/2b, ed. K. Schaifers, \& H. H. Voigt (Springer Berlin, Heidelberg, New York), 451

Shao, M., Colavita, M. M., Hines, B. E., et al. 1988, A\&A, 193, 357

Tsuji, T. 1981, A\&A, 99, 48

Tuthill, P. G., Haniff, C. A., \& Baldwin, J. E. 1997, MNRAS, 285,529

Weigelt, G., Balega, Y., Blöcker, T., et al. 1998, A\&A, 333, L51

Wilson, R. W., Baldwin, J. E., Buscher, D. F., \& Warner, P. J. 1992, MNRAS, 257, 369

Wittkowski, M., Langer, N., \& Weigelt, G. 1998, A\&A, 340, L39

Young, J. S., Baldwin, J. E., Boysen, R. C., et al. 2000, MNRAS, 315, 625 